\title{
Combination of remote sensing and kriging to improve soil salinity mapping in the Hmadna plain (Algeria)
}

\author{
Abdelkader Douaoui *, Ibrahim Yahiaoui \\ University of Khemis Miliana, Laboratory Crop Production and Sustainable Valorisation of the Natural Resources, Algeria \\ *Corresponding author e-mail : aekdouaui@gmail.com \\ Received : 13.12 .2013 \\ Accepted: 14.04 .1014
}

\section{Abstract}

The plain of Hmadna is located in the western part of the plains of Cheliff (North-western of Algeria) with a surface of more than 10,000 ha. The salinity mapping at the scale of whole plain is difficult to be performed because it would be necessary to collect and analyse a large number of samples in order to reach a good spatial estimate. The satellite's remote-sensing capability, with the quantity of information which it offers and its broad field of view, seems to be the most suitable tool to chart salinity, when the spectral data are combined with a reduced number of soil samples., The necessary precondition for the operational use of this technique is the existence of a good correlation between the data measured directly from soil samples with that resulting from the remote-sensing. The objective of this work is to show the importance of using optical sensors with a very high spatial and spectral resolution in the improvement of the mapping surface layer soil salinity. In the first part of this paper we develop a relationship between the remote-sensed data (very high resolution) and the measured salinity of ground samples (as expressed by sample electrical conductivity (EC), through statistical analysis (Ordinary Kriging) to create a salinity index just in the sample area for this dataset. Then in the second part of the paper, we apply this developed relationship to the whole of the WorldView-2 image set, to extrapolate a soil salinity map for the whole area.

Keywords: Salinity, remote sensing, geostatistics, GIS

\section{INTRODUCTION}

The mapping of salinity at the whole plain scale is difficult to establish because it is necessary to take and analyze a big number of samples in order to reach a good spatial estimate. The satellite's remote - sensing, with the quantity of information that it provides and its importance in the study field, seems finally to be the most suitable tool to map salinity with a reduced number of samples. Meanwhile, the necessary precondition within its operational use is the existence of a good correlation between the measured data directly from the soil and those resulting from the remote-sensing process [1].

The objective of this work is to show the importance of using optical sensors with a very high spatial resolution and high spectral resolution in the improvement of the soil salinity mapping of surface layers. In a first part, we have confronted the digital data of remote-sensing resulted from the WorldView-2 image to the Geostatistical analyzes (ordinary Kriging) mapped from the data of salinity measured from soil samples, only on the level of the sampled zone. The last part will consist in extrapolating the mapping of salinity to the whole satellite image by spatial estimate of salinity starting from the relation determined between measured salinity and remote-sensing data for the plain of Hmadna (Algeria).

\section{MATERIALS AND METHODS}

\section{Sampling and Satellite imagery Information}

The recognition of the soil and samples selection were obtained during the month of June, 2010. The choice of this period coincides with a very weak vegetation cover (even non-existent) in the sampled areas of the perimeter of Hmadna where irrigated surfaces are absent. The accumulation of salts is also more important there on soil surface and consequently more easily detectable, even more with the presence of a sebkha on the surface where salts is accumulated on surface during this 
period. The salinity of the 122 sampled points was measured in the laboratory by the method of the saturated extract and the adopted sampling is of laminated type (semi-random) (fig. 1A).

We used a scene from the WorldView-2 satellite taken in June 2010 (fig. 1B). It has 8 bands with very high spatial resolution $(1,8 \mathrm{~m})$ also a high spectral resolution distributed between the visible and the near infra red

- The visible with: Coastal Blue (400-450 nm) Blue (450-510 nm) - Green (510-580 nm) - Yellow (585-625 nm) - Red (630-690 nm) - Red-Edge (705$745 \mathrm{~nm})$.

- The Near Infra Red: NIR1 (770-895 nm) - NIR2 (860-1040 nm).

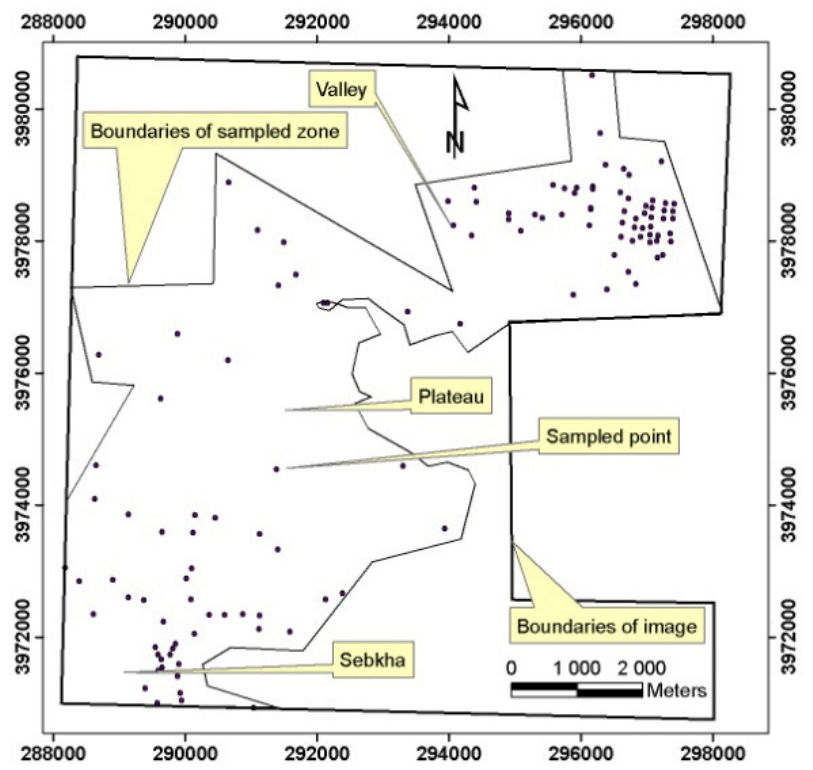

(A)
$\mathrm{Z}^{*}\left(\mathrm{x}_{0}\right)=\sum_{i=1}^{n} \lambda_{i} Z\left(\mathrm{x}_{i}\right) \quad \sum_{i=1}^{n} \lambda_{i}=1$

Where $\mathrm{n}$ is the number of neighbours considered and $\lambda_{i}$ are the weights derived from variogram fitting.

\section{Regression-kriging (RK)}

Spatial predictions using regression-kriging are obtained by:

$Z * R K\left(x_{0}\right)=f\left[R S m o d\left(x_{0}\right)\right]+\Sigma \lambda i \operatorname{ERs}\left(x_{0}\right)$

Where $f\left[R S m o d\left(x_{0}\right)\right]$ is the regression model between electrical conductivity and the index derived from remote-sensing data at $x_{0}, E R s\left(x_{0}\right)$ the residuals from regression estimation at $x_{0}$, and $\lambda i$ the weights determined by ordinary kriging of the residuals [2].

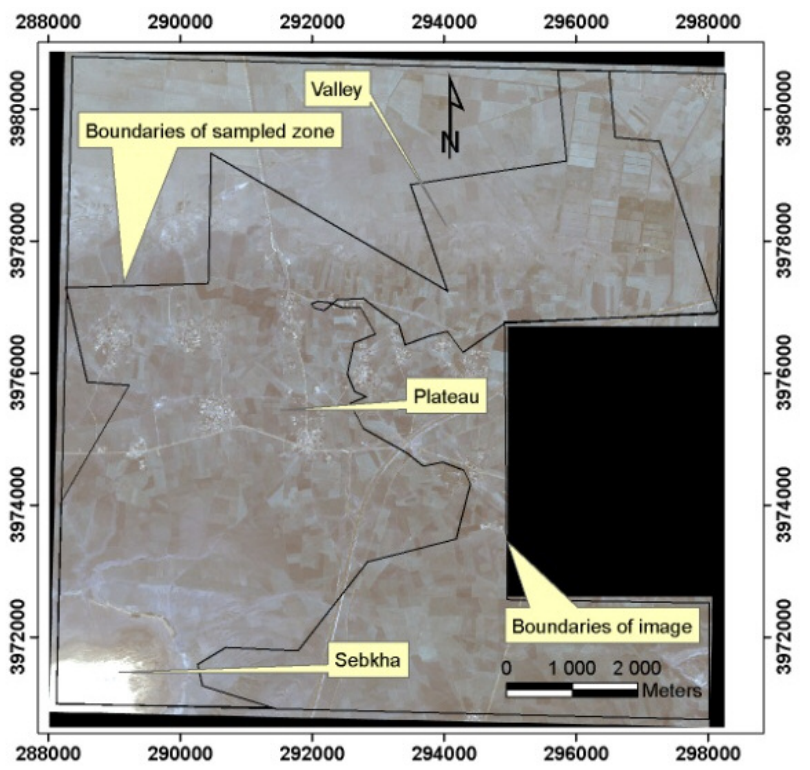

(B)

Figure 1. Plan of the soil points sampling (A), The WorldView-2 scene (June 2010) (B)

\section{Spatial prediction of salinity}

The electrical conductivity observations are denoted $Z\left(x_{1}\right), Z\left(x_{2}\right), \ldots, Z(x i)$, where $x i$ is a location vector and $i$ the number of observations used for prediction purposes. We used of two types of prediction techniques to estimate the $Z^{*}\left(x_{0}\right)$ property at any new unvisited location $\left(x_{0}\right)$ on a $2 \times 2 \mathrm{~m}$ grid laid out over the Hmadna plain.

\section{Ordinary kriging (OK)}

Ordinary kriging with a global variogram (OKGV) was used as a basis of comparison with regression kriging, as predictions may only be derived from ground measurements:

\section{RESULTS AND DISCUSSION}

\section{Salinity index calculation}

The good significant correlations between the bands of the satellite image and the measured salinity data led us to seek the best possible combination between the various bands and salinity. After having started with those already existed in bibliography, we sought to determine our own index.

We find several indices in the bibliography which are used to estimate salinity from the satellite image data. Among those, some can be applied to a WorldView-2 image, we have: 
- Soil salinity index: $\mathrm{SI}=\left(\mathrm{Blue}^{2}{ }^{*} \text { Rouge }^{2}\right)^{0,5},[3]$.

- Normalized Differential Salinity Index (NDSI); $\mathrm{NDSI}=($ Red-NIR $) /($ Red+NIR), NIR : Near Infra Red, [4].

- Salinity index (IS): IS = $(\text { Red + NIR })^{0,5},[2]$;

The vegetation indices such as the NDVI I=(NIRRed)/(Red+NIR), and brightness index $\left(\mathrm{IB}=\left(\text { Rouge }^{2}+\mathrm{NIR}^{2}\right)^{0,5}\right)$ are also used in the estimate of salinity [5].

Table $n^{\circ} 3$ shows that the strongest correlation corresponds to the salinity index of Hmadna (SIh) where we found a coefficient of correlation equalizes to 0.86 for the WorldView-2 image. This index equation is:

Slh $=(\text { (Coastal Blue })^{2}-50 *($ Green+Yellow+NIR2 $\left.)\right)^{0.5}$ about the spatial structure of the studied variable. The variogram of the measured EC shows the existence of a good structure with an average variogram adjusted with a spherical function with a null effect of nugget, a stage $=8000(\mathrm{dS} / \mathrm{m})^{2}$ and a range $=2800 \mathrm{~m}$.

The spatial estimate of salinity was made by ordinary kriging (OK) on the sampled zone (fig. 3A). The salinity map already established shows the existence of an excessively high salinity (EC > 50 $\mathrm{dS} / \mathrm{m}$ ) in the sebkha and its neighbourhoods, a variable salinity in the plain and a low salinity on the mid-hill [6].

Table 1. Coefficients of correlation between measured salinity and remote-sensing indices.

\begin{tabular}{lllllll}
\hline & $\mathrm{IB}$ & $\mathrm{NDVI}$ & $\mathrm{NDSI}$ & $\mathrm{SI}$ & $\mathrm{IS}$ & SIh \\
\hline EC & 0,35 & $-0,57$ & 0,57 & 0,55 & 0,46 & 0,86 \\
\hline
\end{tabular}

In thick, significant values at the threshold alpha $=0,050$ (bilateral test)

The linear regression established between the measured EC and the SIh index shows a good linear relation between the soil data and those of the remote-sensing (fig. 2). This level of correlation is largely sufficient to use remote-sensed data as an auxiliary variable in the spatial estimate and the mapping of salinity.
Salinity map estimated by the regression kriging method from the satellite image

The creation of a new channel helped to transform the original satellite image of 08 bands into a new image by the combination of several bands according to the equation resulted from the

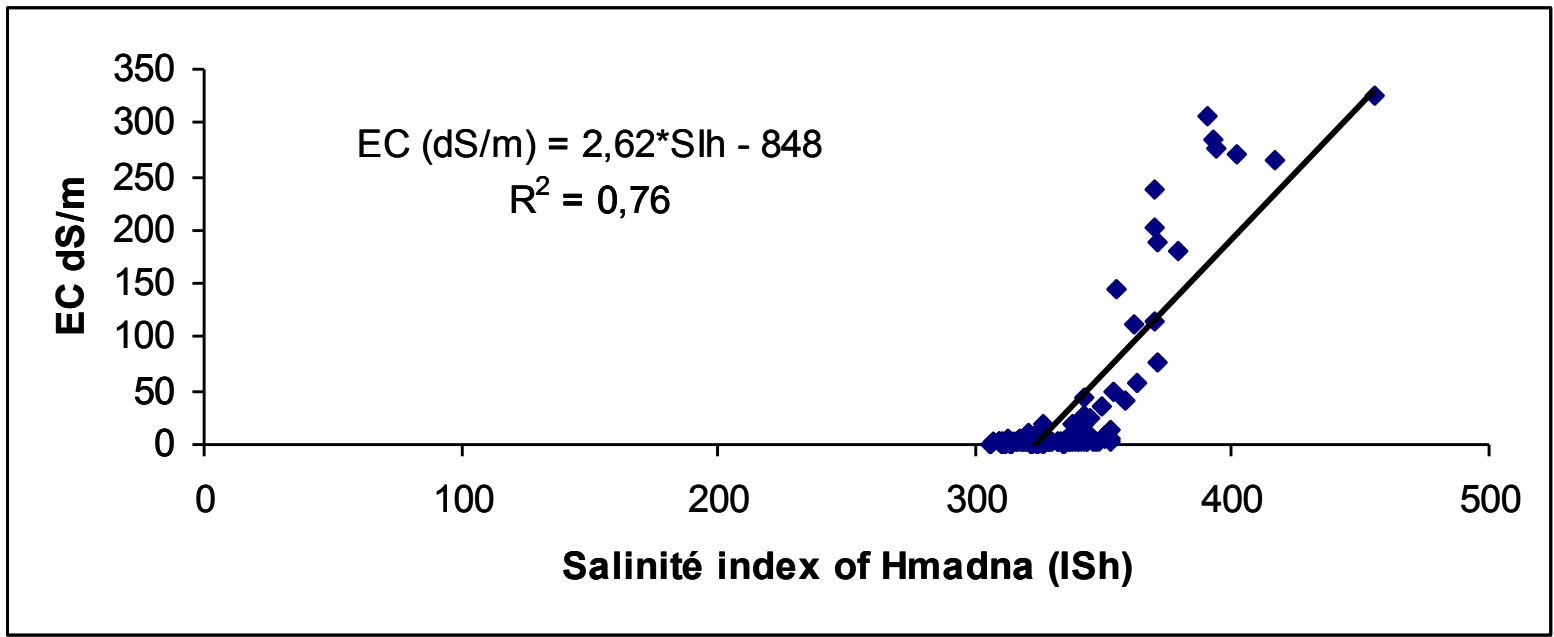

Figure 2. Linear regression between measured salinity $(E C)$ and the salinity index of Hmadna)

\section{Mapping of salinity}

Salinity map estimated by ordinary kriging on the measured salinity data

The application of the kriging requires the establishment of the variogram which informs us
SIh index. This image containing the data of remote-sensing was also transformed by the linear regression equation between the measured EC and the salinity index SIh.

The application of the regression kriging method requires the presence of a spatial 


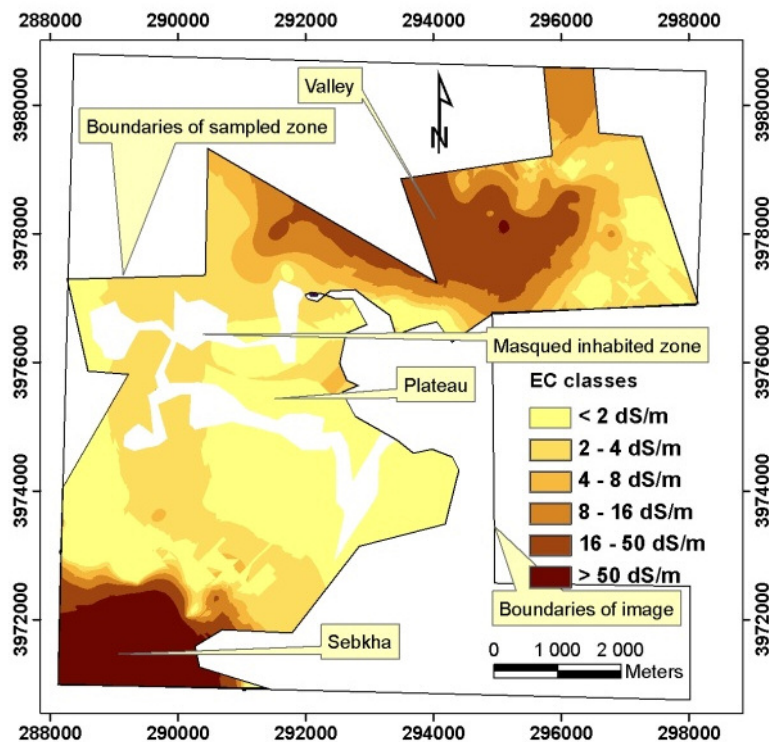

(A)

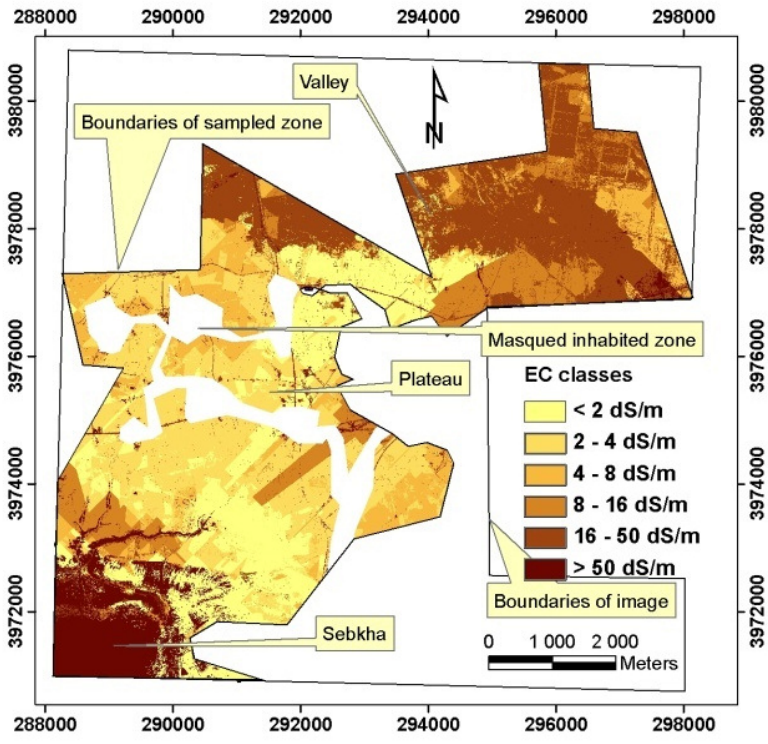

$(\mathrm{A})$
Figure 3. Salinity map estimated by ordinary kriging $(\mathrm{A})$ and regression-kriging $(\mathrm{B})$

continuity of the residues which are calculated between the data of measured EC and the data of estimated EC from the salinity index SIh. The variogram established for the residues of the EC shows a continuity and a spatial regularity of the residues; it is adjusted with a spherical function, an effect of nugget $=10(\mathrm{dS} / \mathrm{m})^{2}$, a stage $=1700$ $(\mathrm{dS} / \mathrm{m})^{2}$ and a range $=900 \mathrm{~m}$.

The salinity map estimated by kriging regression shows the same spatial distribution of salinity as the estimated map by ordinary kriging with, however, higher salted surfaces in the plain(fig. 3B).

Comparison between the salinity map of remote-sensing and ordinary Kriging

The comparison of the surfaces by class of salinity (tab. 2) made by the two methods (OK and RK) give different results. The notable difference is on the level of the class surface representing the not or moderate salted soils where the surface obtained is lower for the regression-kriging approach. However, in the case of high salinity, the percentage surface is higher this time with the regression-kriging approach, which reduces the errors on the surfaces estimated between the two highest levels: high salted ( $16<\mathrm{EC} 50 \mathrm{dS} / \mathrm{m})$ and very high salted (EC $50 \mathrm{dS} / \mathrm{m})$.

The validation of the estimates for the two methods was done by 15 points of sampled and measured EC at the same time as the 120 points used in the mapping. The criteria of selected validation confirm what was found in the calculation of the surfaces and witnessed by the benefit in the quality of the estimates through a reduction of the under estimation errors (tab. 3).

Table 2. Classes surface of estimated EC by remote-sensing and ordinary Kriging.

\begin{tabular}{|c|c|c|c|c|c|c|c|}
\hline \multicolumn{8}{|c|}{ Classes de CE (dS/m) } \\
\hline & $<2$ & $2-4$ & $4-8$ & $8-16$ & $16-50$ & $>50$ & Total \\
\hline \multicolumn{8}{|c|}{ EC estimed by ordinary kriging } \\
\hline Ha & 1300,64 & 1053,67 & 342,81 & 389,64 & 452,1 & 295,14 & 3834 \\
\hline$\%$ & 34 & 27 & 9 & 10 & 12 & 8 & 100 \\
\hline \multicolumn{8}{|c|}{ EC estimed by regression-kriging } \\
\hline Ha & 738,99 & 913,96 & 493,89 & 426,46 & 874,17 & 386,53 & 3834 \\
\hline$\%$ & 19 & 24 & 13 & 11 & 23 & 10 & 100 \\
\hline
\end{tabular}

Table 3. Statistical validation of estimation quality

\begin{tabular}{cccc}
\hline & ME & RMSE & RMSSE \\
\hline KB & -0.16 & 8,12 & 1,18 \\
RK & 0,21 & 8,34 & 0,98 \\
\hline
\end{tabular}




\section{CONCLUSIONS}

The salinity map obtained by WorldView-2 image revealed a good correlation compared to the Geostatistical processing of the collected data directly from the ground. The same spatial distribution of various levels of salinity was confirmed by introducing the salinity index SIh, which corresponds to exogenous information derived from WorldView2 image, it is possible to establish salinity maps with greater accuracy by using ordinary kriging. The regression-kriging (RK) method leads to a relatively high level of accuracy in the spatial estimation of salinity. The resultant maps are the closest to groundtruth and the surface area estimation is most accurate among the tested methods. This result is very encouraging. It witnesses the possibility of making a regular follow-up of the various levels of the salinity spatial dynamics on a regional scale. This mapping could be generalized to all the plains of Cheliff which make more than 150000 ha with a much reduced cost and a time record by using WorldView-2 image. Indeed, it is not necessary any longer to carry out displacements of great width on the study area, which are inevitably expensive. Finally, several precursory works and these two mapping approaches (remote-sensing and geostatistics) have unfortunately detected an important expansion of salinity. The primary reason is the groundwater irrigation often charged with salt. This risks extending to the untouched soils yet. Concrete measures must be taken quickly to contain this damage. In order to help all the interested parties of this serious problem, the best approach would be to optimize the salinity mapping with a combination of geostatistics and remote-sensing by adopting a better sampling strategy.

\section{Acknowledgements}

The authors wish to acknowledge to DGAPAUNAM (Direccion General de Asuntos del Personal Académico of UNAM) for the support received through the PAPIIT project IN221613-3.

\section{REFERENCES}

[1] G. I. Metternicht, J. A. Zinck, 2003. Remote sensing of soil salinity: potentials and constraints'. Remote Sensing of Environment, 85(1), 1-20.

[2] A. Douaoui, H. Nicolas, Ch.Walter, 2006. Detecting salinity hazards within a semiarid context by means of combining soil and remote-sensing data'. Geoderma, 134(1-2), 217-230.

[3] N. M. Khan, V. Rastoskuev, E. Shalina, Y. Sato, 2001. 'Mapping salt-affected soil using remote sensing indicators. A simple approach with the use of Gis Idrissi'. 22nd Asian Conference on Remote Sensing, 5-9 November, Singapore.

[4] F. Al-Khaier, 2003. Soil Salinity Detection Using Satellite Remote Sensing. ITC, The Netherlands, Master of Science, 61 p.

[5] A. Fernadez-Buces, C. Sieb, S. Cram, J. L. Palacio, 2006. Mapping soil salinity using a combined spectral response index for bar soil and vegetation : A case study in the former lake Texcoco, Mexico. Journal of Arid Environments. 65, 644-667.

[6] A. Douaoui, Ph. Lépinard, 2010. Télédétection et salinité, Cartographie de la salinité des sols de la plaine algérienne du Bas-Chéliff. Géomatique expert, N76, aout et septembre, 36-41. 